\title{
Bill R. Appleton Receives 1995 Woody Award
}

Bill R. Appleton of Oak Ridge National Laboratory (ORNL) recently received the 1995 Woody Award "in recognition of outstanding service and dedication, on behalf of MRS, as exemplified by Woody White as president in 1984."

Outgoing MRS president Julia Phillips presented the award during the 1995 MRS Fall Meeting Council Dinner on December 1, 1995.

Appleton's previous MRS involvement includes service as a meeting chair in 1983, as second vice-president in 1984, and as a three-time councillor with terms beginning in 1985, 1988, and 1993. He has also been a member of the Public Affairs, External Affairs, Long-Range Planning, and Program Committees, as well as being active in the International Union of Materials Research Societies.

"On many occasions Bill Appleton has made the right connections to greatly strengthen the society's public affairs efforts," said Tom Picraux, chair of the MRS Public Affairs Committee. Picraux cited Appleton as a key force in connecting MRS to the national Advanced Materials and Processing Program in the late 1980s, which "raised MRS to a new level of national visibility."

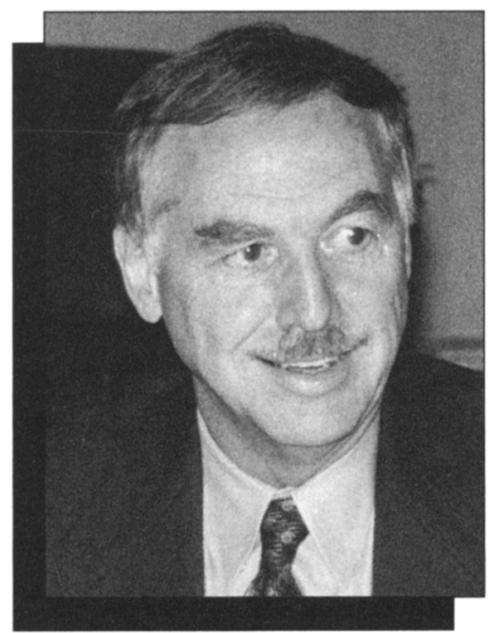

Bill R. Appleton

While chair of the Solid State Sciences Committee, which coordinated the National Research Council study Materials Science and Engineering for the 1990s, Appleton served as designated editor of the MRS publication Communications on the Materials Science and Engineering Study. This book provided input to the NRC study from MRS members and meeting attendees.
Woody White, the award's namesake, spoke highly of Appleton's MRS involvement, "At ORNL he has encouraged others to become active in the society. My own participation in MRS would not have been possible without his support and encouragement while I was a member of his group at ORNL."

Appleton is vice president of Lockheed Martin Energy Research Corporation and associate laboratory director for advanced materials, physical, and neutron sciences at ORNL where he manages the Chemical and Analytical Sciences, Metals and Ceramics, Physics, Research Reactors, and Solid State divisions, and the Neutron Sciences Program. His work has focused on fundamental research in the use of ion-beam and laser processing techniques for surface modification of materials, and on fundamental studies of ion-solid interactions.

Commenting on his long-time service to MRS, Appleton said, "The Materials Research Society has responded to a scientific need, which is one of the reasons why it has done so well. I have thoroughly enjoyed seeing it grow to become such an effective organization."

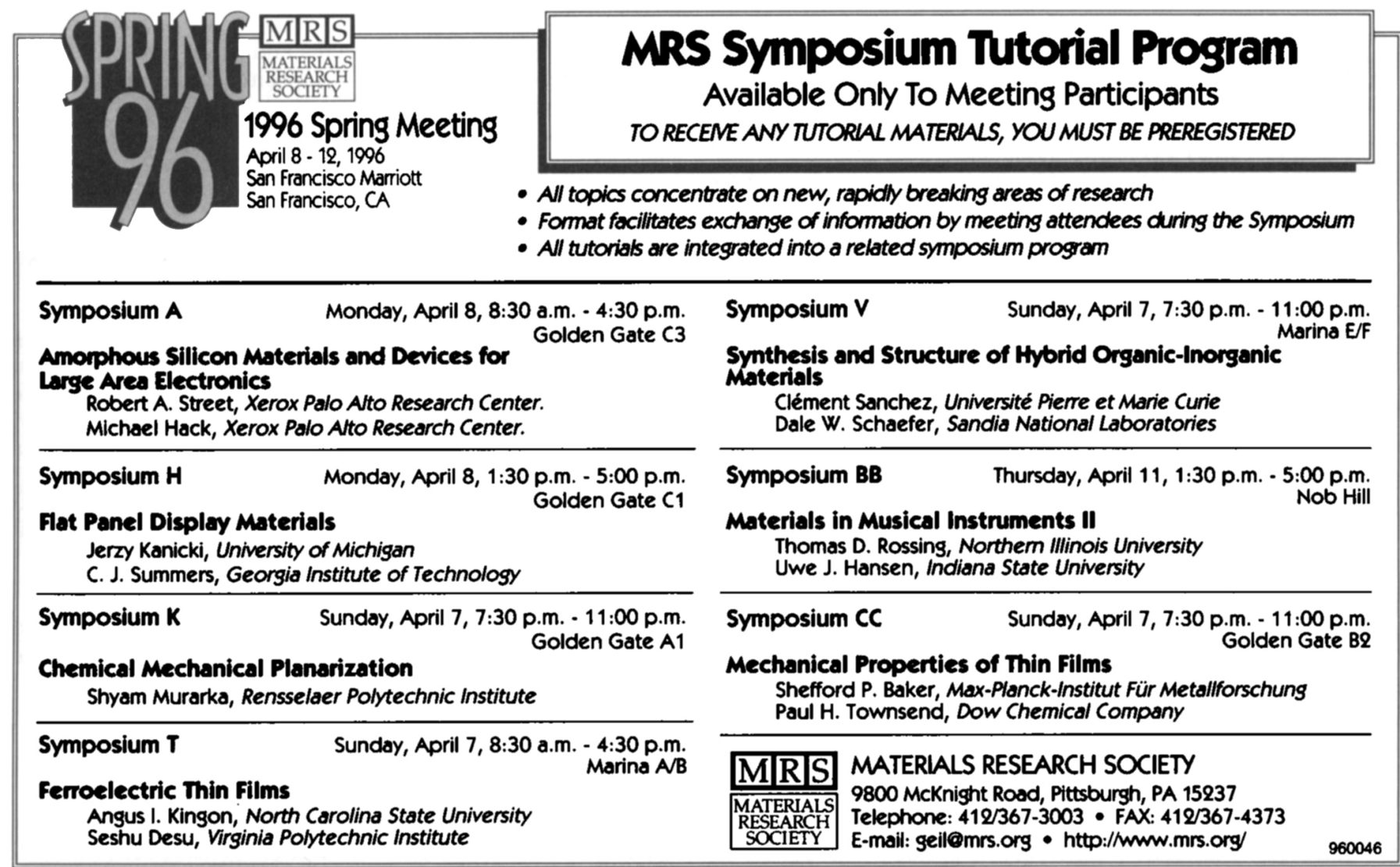




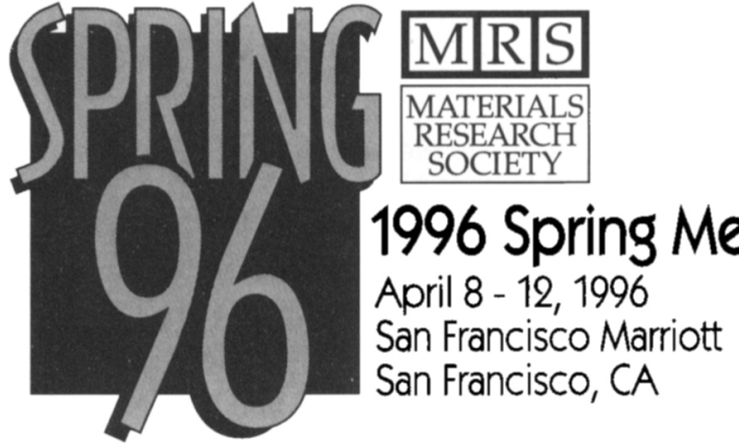

\section{TECHNICAL PROGRAM}

A: Amorphous Silicon Technology - 1996

B: Defects and Interfaces in Lattice-Mismatched Semiconductor Heterostructures

C: Compound Semiconductor Electronics and Photonics

D: Rare-Earth Doped Semiconductors II

E: III-Nitride, $\mathrm{SiC}$, and Diamond Materials for Electronic Devices

F: $\quad$ GeSi and Related Compounds

G: Semiconductors on Insulators - Fundamentals and Technology

$\mathrm{H}$ : $\quad$ Flat Panel Display Materials II

I: Liquid Crystals for Advanced Technologies

J: Thin Films for Photovoltaic and Related Device Applications

K: Advanced Metallization for Future ULSI

L: Materials Reliability in Microelectronics VI

M: Materials and Processes for Peripheral Microelectronic Devices

N: Rapid Thermal and Integrated Processing V

O: Microwave Processing of Materials V

P: $\quad$ Microporous and Mesoporous Materials

Q: Materials Challenges for Applications of High- $\mathrm{T}_{\mathrm{C}}$ Superconductors

R: Fiber Materials for Electronics, Optoelectronics, and Sensors

S: Aqueous Chemistry and Geochemistry of Oxides, Oxyhydroxides, and Related Materials

T: $\quad$ Ferroelectric Thin Films V

U: Layered Materials for Structural Applications

V: Better Ceramics through Chemistry VII - Organic/Inorganic Hybrid Materials

W: Computational Materials Science - Structural, Mechanical, and Transport Properties

X: Frontiers of Materials Research

Y: $\quad$ Structure-Controlled Macromolecules of Nanoscopic Dimensions

Z: Environmentally Degradable Polymers

AA: Innovations in Instrumentation for Materials Research

BB: Materials in Musical Instruments II

CC: Thin Films - Stresses and Mechanical Properties VI

DD: Applications of Synchrotron Radiation to Materials Science III

\section{MRS TUTORIAL PROGRAM}

Half-day tutorial sessions immediately preceding the first day of selected symposia will concentrate on new, rapidly breaking areas of research. The new tutorials are designed to encourage the exchange of information among meeting attendees during the symposium.

Meeting attendees interested in attending the tutorials must preregister for them. See details on the MRS Homepage on the World Wide Web (http://www.mrs.org).

A key forum for discussion of

interdisciplinary leading edge materials

research from around the world.

A variety of meeting formats -

oral, poster, round-table, forum and

workshop sessions maximize

participation.

\section{EXHIBIT}

A complimentary exhibit will be held in conjunction with the MRS 1996 Spring Meeting in San Francisco with companies displaying analytical and processing equipment and services closely paralleling the nature of the technical symposia. The exhibit will be conveniently located near the technical session rooms, and the program will be arranged to allow participants ample time to visit the exhibit.

Companies interested in exhibiting should contact: Mary E. Kaufold, Manager, Advertising and Exhibits, Materials Research Society, 9800 McKnight Road, Pittsburgh, PA 15237 USA; Telephone (412) 367-3036; Fax (412) 367-4373; E-mail: kaufold@mrs.org

\section{PROCEEDINGS}

Many symposia from this meeting will publish refereed proceedings volumes. MRS members and meeting attendees may purchase copies of these proceedings at special prepublication prices and receive priority shipment upon publication. To take advantage of these special prices, order your proceedings while registering for the meeting. For information on nonmember proceedings prices, Telephone (412) 367-3004, ext. 558; Fax (412) 367-4373.

\section{SYMPOSIUM AIDE OPPORTUNITIES}

Graduate students who plan to attend the 1996 Spring Meeting can earn MRS membership benefits and offset travel expenses by participating in the meeting as Symposium Aides. As an aide, the student will receive a waiver of the student registration fee, complimentary membership (July 1, 1996, through June 30, 1997), and a small stipend toward expenses after assisting in four full half-day sessions. For further information or to request an application form, contact MRS Headquarters, Telephone (412) 367-3003; Fax (412) 367-4373; E-mail: info@mrs.org.

\section{JOB PLACEMENT}

A confidential placement service will be offered during the Meeting to MRS members and meeting registrants. Individuals using the Job Placement interviewing services at the MRS Spring Meeting must register for both the meeting and Job Placement Service. However, you need not attend the meeting to have your résumé included on file.

For further information, consult the MRS Homepage on the World Wide Web (http://www.mrs.org) or inquire via e-mail (info @mrs.org); Telephone (412) 367-3003; Fax (412) 367-4373.

To request a 1996 Spring Meeting program book or further information on any meeting activities, contact:

\section{MATERIALS RESEARCH SOCIETY}

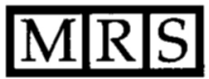

MATERIALS

RESEARCH

SOCIETY
9800 Mcknight Road

Pittsburgh, PA 15237-6006

Telephone (412) 367-3003

Fax (412) 367-4373

info@mrs.org 

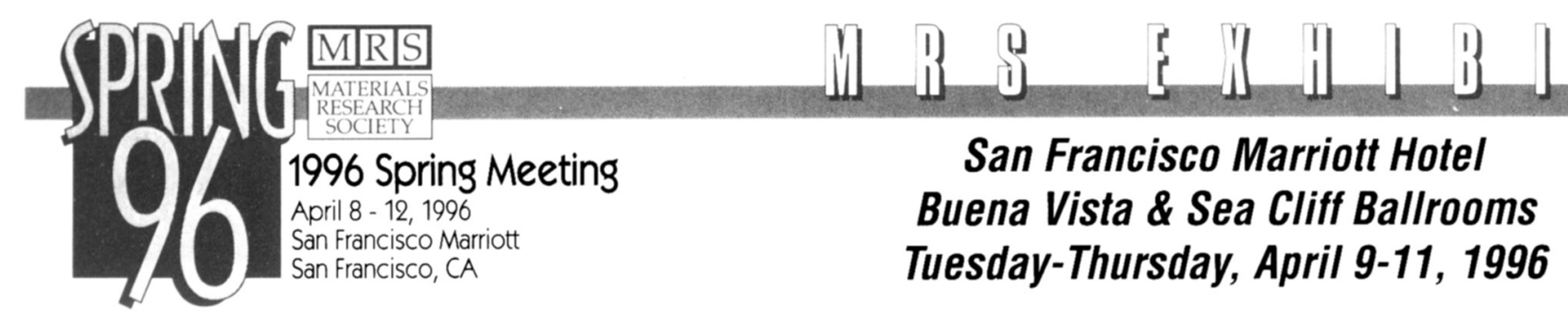

The MRS Exhibit, to be held in conjunction with the 1996 MRS Spring Meeting, will encompass the full spectrum of equipment, products, software, publications and services for materials research. As always, the exhibit will closely parallel the nature of the technical symposia. The technical program has been arranged to allow meeting participants ample opportunity to visit the exhibit, and MRS encourages attendees to visit the exhibit by scheduling coffee breaks, deli-style lunches, and a meeting-wide reception in the exhibit hall.

\section{Partial list of 1996 Spring Exhibitors:}

(As of January 1996)

ABB Extrel

Academic Press, Inc.

Advanced Technology Materials, inc.

Aetrium

AIXTRON

AJA International

American Institute of Physics

A\&N Corporation

APD Cryogenics, Inc.

ASTEX/Applied Science \& Technology, Inc.

Axic, Inc.

Bede Scientific

BIOSYM/Molecular Simulations

Blake Industries, Inc.

Burleigh Instruments

Cabot Corporation

Ceramaseal

CHA Industries

Chemat Technology, Inc.

Commonwealth Scientific Corporation

Continental Electronics Corporation

Cressington Scientific Instruments, Inc.

Cryomech, Inc.

CVC Products

CVD Products, Inc.

Denton Vacuum, Inc.

Depths of the Earth Company, L.C.C.

Digital Instruments

Duniway Stockroom Corporation

Elsevier Science, Inc.

EMCORE Corporation

ERG Inc.

Charles Evans \& Associates

FEI Company

E.A. Fischione Instruments, Inc.

Fisons Instruments

Gatan, Inc.

Goodfellow Corporation

Heraeus Amersil, Inc.

High Voltage Engineering Europa

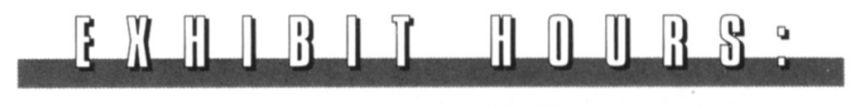

Tuesday, noon - 7:00 p.m.

Complimentary Reception 5:00 - 7:00 p.m.

Wednesday, 9:30 a.m. - 5:00 p.m.

Thursday, 9:30 a.m. - 2:00 p.m.
Hi-Tech Ceramics

Huntington Mechanical Labs., Inc.

Hysitron, Inc.

IBM Analytical Services

Instruments SA, Inc.

Intelligent Sensor Technology, Inc.

Ion Tech, Inc.

IOP Publishing, Inc.

Isoflux, Inc.

JEOL USA, Inc.

Keithley Instruments

Kevex X-Ray

Kluwer Academic Publishers

Lake Shore Cryotronics, Inc.

Lambda Technologies, Inc.

Lasertec U.S.A., Inc.

Kurt J. Lesker Co.

LUXTRON Corporation

Magnet Sales \& Manufacturing

MARCH Instruments, Inc.

Materials Research Group, Inc.

MDC Vacuum Products Corp.

Melles Griot

Micramics, Inc.

Micro Photonics, Inc.

MMR Technologies, Inc.

Modular Process Technology Corp.

MTS Systems Corp.

MVSystems, Inc.

$n \&$ k Technology, Inc.

Nano Instruments, Inc.

National Electrostatics Corp.

New Focus, Inc.

Nor-Cal Products, Inc.
Olympus America, Inc.

Omicron Associates

Oxford Applied Research

Park Scientific Instruments

Philips Electronic Instruments Co.

Philips Semiconductors/Materials

Analysis Group

Photonetics Inc.

Physical Electronics

Plasma Sciences, Inc.

Pure Tech, Inc.

Quesant Instrument

Radiant Technologies

Research and PVD Materials Corporation

Rigaku/USA, Inc.

RJ Lee Instruments, Ltd.

Schumacher

SKION Corporation

South Bay Technology, Inc.

Staib Instruments, Inc.

SURFACE

Surface/Interface, Inc.

SVT Associates, Inc.

Technical Instruments, Inc.

Tencor Instruments

Thermionics Laboratory, Inc.

Union Carbide Crystal Products

US Thin Films Products, Inc.

VCR Group, Inc.

Virginia Semiconductor, Inc.

Vital Image Technology, Inc.

Voltaix, Inc.

SEE AD IN THIS ISSUE 

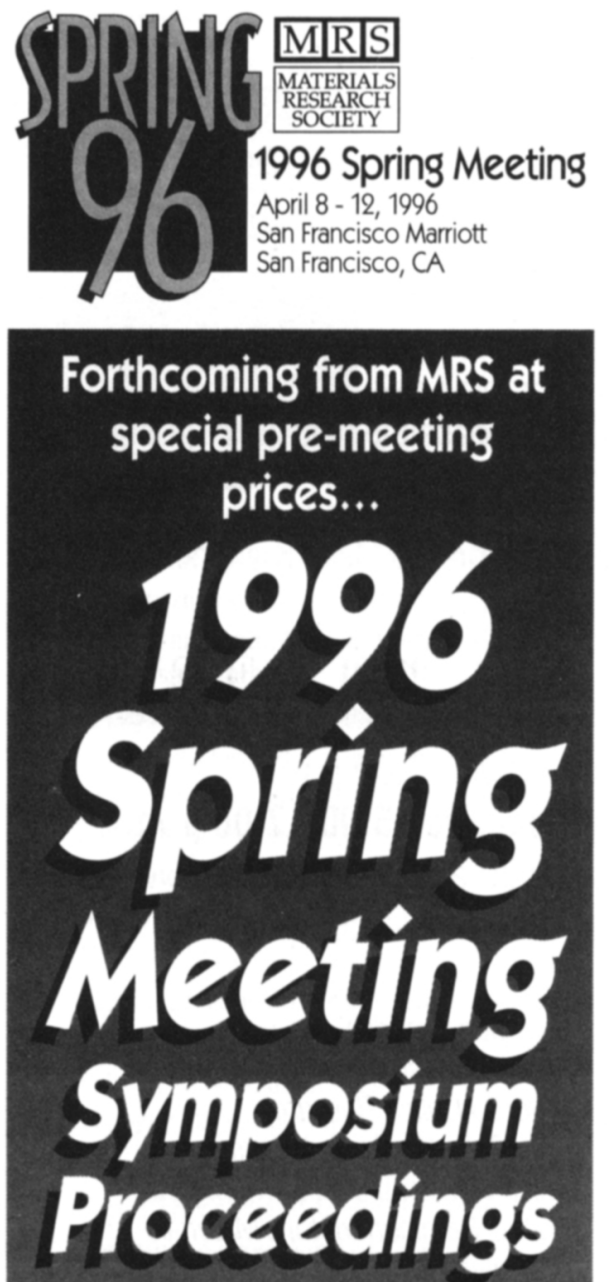

Place your order today for

proceedings of the 1996 MRS

Spring Meeting in San Francisce and SAVE!

\begin{tabular}{|c|c|}
\hline $\mathrm{M} R \mathrm{R}$ & Materials Research Society \\
\hline $\begin{array}{l}\text { MATERIALS } \\
\text { RESEARCH } \\
\text { SOCIETY } \\
\end{array}$ & $\begin{array}{l}9800 \text { McKnight Road, Pittsburgh, PA } 15237 \\
\text { Phone: 412-367-3012, Fax: 412-367-4373 }\end{array}$ \\
\hline $\begin{array}{l}\text { In Europe, } \\
\text { Clarke Asso } \\
\text { Fourth Floo } \\
\text { Queen Cha } \\
\text { Bristol BS1 } \\
\text { ENGLAND } \\
\text { Phone: } 011 \\
\text { Fax: } 011\end{array}$ & $\begin{array}{l}\text { Africa and the Middle East: } \\
\text { ciates-Europe, Ltd. } \\
\text { The Rackhay } \\
\text { lotte Street } \\
\text { HJ } \\
99268864 \\
9226437\end{array}$ \\
\hline
\end{tabular}

A: Amorphous Silicon Technology-1996 Editors: M. Hack, E.A. Schiff, S. Wagner, A. Matsuda,

R. Schropp

ISBN: $1-55899-323-1 \quad$ Code: $420-B$

$\begin{array}{lll}\$ 55.00 & \$ 65.00 & \text { MRS Member } \\ \$ 65.00 & \$ 74.00 & \text { U.S. List } \\ \$ 76.00 & \$ 85.00 & \text { Non-U.S. }\end{array}$

C: Compound Semiconductor Electronics and Photonics Editors: R.J. Shul, S.J. Pearton, C-S. Wu, F. Ren ISBN: $1-55899-324-X \quad$ Code: $421-B$
$\$ 55.00 \quad \$ 65.00$
$\$ 65.00 \quad \$ 74.00$
U.S. List
$\$ 76.00$
$\$ 85.00$
Non-U.S.

MRS Member

D: Rare-Earth Doped Semiconductors II

Editors: A. Polman, S. Coffa, R. Schwartz

ISBN: 1-55899-325-8 Code: 422-B

$\begin{array}{lll}\$ 52.00 & \$ 61.00 & \text { MRS Member } \\ \$ 61.00 & \$ 70.00 & \text { U.S. List } \\ \$ 72.00 & \$ 81.00 & \text { Non-U.S. }\end{array}$

E: III-Nitride, SiC, and Diamond Materials for

Electronic Devices

Editors: D.K. Gaskill, C. Brandt, R.J. Nemanich

ISBN: 1-55899-326-6 Code: 423-B

$\begin{array}{lll}\$ 55.00 & \$ 65.00 & \text { MRS Member } \\ \$ 65.00 & \$ 74.00 & \text { U.S. List } \\ \$ 76.00 & \$ 85.00 & \text { Non-U.S. }\end{array}$

H: Flat Panel Display Materials II

Editors: M. Hatalis, J. Kanicki, C.J. Summers, F. Funada ISBN: 1-55899-327-4

$\begin{array}{lll}\$ 52.00 & \$ 61.00 & \text { MRS Member } \\ \$ 61.00 & \$ 70.00 & \text { U.S. List } \\ \$ 72.00 & \$ 81.00 & \text { Non-U.S. }\end{array}$

I: Liquid Crystals for Advanced Technologies

Editors: T.J. Bunning, S.H. Chen, W. Hawthorne,

N. Koide, T. Kajiyama

ISBN: 1-55899-328-2

$\begin{array}{lll}\$ 55.00 & \$ 65.00 & \text { MRS Member } \\ \$ 65.00 & \$ 74.00 & \text { U.S. List } \\ \$ 76.00 & \$ 85.00 & \text { Non-U.S. }\end{array}$

$\mathrm{J}$ : Thin Films for Photovoltaic and Related Device Applications

Editors: C. Eberspacher, H.W. Schock, D. Ginley,

T. Catalano, T. Wada

ISBN: 1-55899-329-0

$\begin{array}{lll}\$ 52.00 & \$ 61.00 & \text { MRS Member } \\ \$ 61.00 & \$ 70.00 & \text { U.S. List } \\ \$ 72.00 & \$ 81.00 & \text { Non-U.S. }\end{array}$

K: Advanced Metallization for Future ULSI

Editors: K.N. Tu, J.W. Mayer, J.M. Poate, L.J. Chen

ISBN: $1-55899-330-4 \quad$ Code: 427-B

$\begin{array}{lll}\$ 56.00 & \$ 66.00 & \text { MRS Member } \\ \$ 66.00 & \$ 76.00 & \text { U.S. List } \\ \$ 78.00 & \$ 88.00 & \text { Non-U.S. }\end{array}$

L: Materials Reliability in Microelectronics VI

Editors: W.F. Filter, J.J. Clement, A.S. Oates,

R. Rosenberg, P.M. Lenahan

ISBN: 1-55899-331-2

$\begin{array}{lll}\$ 56.00 & \$ 66.00 & \text { MRS Member } \\ \$ 66.00 & \$ 76.00 & \text { U.S. List } \\ \$ 78.00 & \$ 88.00 & \text { Non-U.S. }\end{array}$

N: Rapid Thermal and Integrated Processing V

Editors: A. Fiory, F. Roozeboom, J.C. Gelpey, M. Öztürk,

R.P.S. Thakur

ISBN: 1-55899-332-0

$\begin{array}{lll}\$ 52.00 & \$ 61.00 & \text { MRS Member } \\ \$ 61.00 & \$ 70.00 & \text { U.S. List } \\ \$ 72.00 & \$ 81.00 & \text { Non-U.S. }\end{array}$

0: Microwave Processing of Materials V

Editors: M.F. Iskander, E.R. Peterson, J.O. Kiggans,

J.Ch. Bolomey

ISBN: 1-55899-333-9

$\begin{array}{lll}\$ 54.00 & \$ 64.00 & \text { MRS Member } \\ \$ 64.00 & \$ 73.00 & \text { U.S. List } \\ \$ 75.00 & \$ 84.00 & \text { Non-U.S. }\end{array}$

P: Microporous and Macroporous Materials

Editors: S.L. Suib, M.E. Davis, S.I. Zones, J.S. Beck,

L.E. Iton, D.R. Corbin, R.F. Lobo

ISBN: $1-55899-334-7 \quad$ Code: $431-B$

$\begin{array}{lll}\$ 54.00 & \$ 64.00 & \text { MRS Member } \\ \$ 64.00 & \$ 73.00 & \text { U.S. List } \\ \$ 75.00 & \$ 84.00 & \text { Non-U.S. }\end{array}$

S: Aqueous Chemistry and Geochemistry of Oxides, Oxyhydroxides, and Related Materials

Editors: J.A. Voigt, B.C. Bunker, W.H. Casey, T.E. Wood, L.J. Crossey

ISBN: 1-55899-335-5

$\begin{array}{lll}\$ 54.00 & \$ 64.00 & \text { Code: } 432-B \\ \$ 64.00 & \$ 73.00 & \text { MRS Member } \\ \$ 75.00 & \$ 84.00 & \text { U.S. List } \\ & \text { Non-U.S. }\end{array}$

T: Ferroelectric Thin Films V

Editors: S.B. Desu, R.E. Jones, R. Ramesh, B.A. Tuttle, I.K. Yoo

ISBN: 1-55899-336-3

$\begin{array}{lll}\$ 54.00 & \$ 64.00 & \text { MRS Member } \\ \$ 64.00 & \$ 73.00 & \text { U.S. List } \\ \$ 75.00 & \$ 84.00 & \text { Non-U.S. }\end{array}$

U: Layered Materials for Structural Applications Editors: J.J. Lewandowski, C.H. Ward, T.F. Broderick, W.H. Hunt, Jr., M.R. Jackson

ISBN: 1-55899-337-1

$\begin{array}{lll}\$ 51.00 & \$ 60.00 & \text { MRS Member } \\ \$ 60.00 & \$ 68.00 & \text { U.S. List } \\ \$ 70.00 & \$ 79.00 & \text { Non-U.S. }\end{array}$

V: Better Ceramics Through Chemistry VIIOrganic/Inorganic Hybrid Materials

Editors: D.W. Schaefer, G.L. Wilkes, C. Sanchez, B. Coltrain

ISBN: 1-55899-338-X

$\begin{array}{ll}\$ 51.00 & \$ 60.00 \\ \$ 60.00 & \$ 68.00 \\ \$ 70.00 & \$ 79.00\end{array}$

Code: $435-B$ MRS Member

U.S. List

Non-U.S.

CC: Thin Films: Stresses and Mechanical Properties VI Editors: W.W. Gerberich, H. Gao, J-E. Sundgren,

S.P. Baker

ISBN: $1-55899-339-8$

$\begin{array}{lll}\$ 51.00 & \$ 60.00 & \text { MRS Member } \\ \$ 60.00 & \$ 68.00 & \text { U.S. List } \\ \$ 70.00 & \$ 79.00 & \text { Non-U.S. }\end{array}$

DD: Applications of Synchrotron Radiation to Materials Science III

Editors: L. Terminello, S. Mini, D.L. Perry, H. Ade

ISBN: $1-55899-340-1 \quad$ Code: $437-B$

$\begin{array}{lll}\$ 56.00 & \$ 66.00 & \text { MRS Member } \\ \$ 66.00 & \$ 76.00 & \text { U.S. List } \\ \$ 78.00 & \$ 88.00 & \text { Non-U.S. }\end{array}$

These books are scheduled for publication by fall or early winter 1996 .

Special pre-meeting prices

(prices on left) effective until April 26, 1996.

After April 26, 1996, pay the higher prices on the right. 